\section{JTI}

JOURNAL OF

TRAUMA AND INJURY

\title{
Comparative Analysis between Spinning and Other Causes in Exercise-Induced Rhabdomyolysis
}

\author{
Do Won Shim, M.D. ${ }^{1}$, Sung Youl Hyun, M.D. ${ }^{2}$, Jae Hyug Woo, M.D. ${ }^{1}$, \\ Jae Ho Jang, M.D. ${ }^{1}$, Jae Yeon Choi, M.D. ${ }^{1}$ \\ Departments of ${ }^{1}$ Emergency Medicine, ${ }^{2}$ Traumatology, Gachon University Gil Medical \\ Center, Gachon University College of Medicine, Incheon, Korea
}

Received: August 30, 2018

Revised: September 29, 2018

Accepted: October 1, 2018

\section{Correspondence to}

Sung Youl Hyun, M.D.

Department of Traumatology, Gachon University Gil Medical Center,

21 Namdong-daero 774beon-gil, Namdong-gu, Incheon 21565, Korea

Tel: $+82-32-460-3015$

Fax: +82-32-460-3019

E-mail: sungyoul@gilhospital.com

Purpose: Spinning-induced rhabdomyolysis (SIR) has been increasing in recent years and accounts for a large proportion of exercise-induced rhabdomyolysis (EIR). The purpose of this study was to compare the clinical features between SIR and non-spinning exercise-induced rhabdomyolysis (NSIR), and to analyze each of these clinical features. Methods: A retrospective chart review was conducted on patients treated due to EIR from January 2006 to March 2018. Patients were divided into the SIR and NSIR groups, and their clinical factors, outcome, and blood chemistries were compared and analyzed. Results: Sixty-two patients were enrolled in this study, with 23 (37.1\%) and 39 (62.9\%) patients categorized in the SIR and NSIR groups, respectively. The SIR group were mostly women $(78.3 \%$ vs. $38.5 \%, p=0.002)$, more frequent EIR occurrence in the first exercise class (60.9\% vs. $15.4 \%, p=0.001)$, and had most complaints of thigh pain $(91.3 \%$ vs. $43.6 \%, p=0.001)$. The SIR group had a higher incidence rate despite its shorter exercise duration ( $90.5 \%$ vs. $62.9 \%, p=0.024)$, longer hospital stay (6.0 [4.5-7.0] vs. 5.0 [3.5-6.0] days, $p=0.080)$, and higher rate of peak CPK (15,000 U/L or higher) $(91.3 \%$ vs. $74.4 \%$, $p=0.182$ ) compared to the NSIR group.

Conclusions: SIR occurs at a higher rate during the first exercise class in women compared to NSIR, and the incidence rate is higher in SIR than in NSIR despite its shorter exercise duration (less than 60 minutes). It is necessary to recognize these risks during spinning exercises and to perform these exercises sequentially and systematically.

Keywords: Rhabdomyolysis; Exercise; Acute kidney injury

This is an Open Access article distributed under the terms of the Creative Commons Attribution Non-Commercial License (http://creativecommons.org/licenses/by-nc/4.0/) which permits unrestricted noncommercial use, distribution, and reproduction in any medium, provided the original work is properly cited. 


\section{INTRODUCTION}

Rhabdomyolysis is a clinical and biochemical syndrome characterized by skeletal muscle necrosis and leakage of intracellular muscle constituents into the circulation [1]. Common initial symptoms of rhabdomyolysis involve generalized myalgia with or without muscle weakness and dark-urine secondary to myoglobin [2]. Multiple etiologies of rhabdomyolysis include burns, crush trauma, muscle compression, muscle overexertion, infections, toxins, drugs, inherited disorders of muscle metabolism, and so on [3]. Among the various etiologies, exercise-induced rhabdomyolysis (EIR) occurs when the energy supplied to the muscle is insufficient [4].

Spinning-induced rhabdomyolysis (SIR) has been increasing in recent years, accounting for a large proportion of EIR cases [5]. Spinning is an indoor cycling exercise that has become popular over the past few years. It is a cardiovascular exercise using stationary cycles with rhythmical music. Although some recent studies have reported on SIR cases [2,5-7], the comparison of the SIR with non-spinning exercise-induced rhabdomyolysis (NSIR) has rarely been studied [8]. Thus, this study aimed to compare the clinical features between SIR and NSIR by analyzing each of their clinical factors, outcomes, and blood chemistries.

\section{METHODS}

\section{Study design and population}

From January 2006 to March 2018, we retrospectively reviewed the records of patients with EIR who were treated in our medical center. The medical center has about 90,000 to 100,000 and $1,100,000$ patients visiting the emergency and outpatient departments annually.

Serum creatine phosphokinase (CPK) value of five times higher than the upper limit of the normal or muscle uptake obtained via whole body bone scan test was a diagnostic method for rhabdomyolysis in previous large studies [8-10]. EIR was defined as a rhabdomyolysis due to marked exertion by trained or untrained people [6]. In this study, we enrolled EIR patients whose serum CPK level was 1,500 U/L or higher. The patients with EIR was divided into the following two groups according to the exercises performed: SIR group for spinning and NSIR group for other exercises.

The diagnosis of acute kidney injury (AKI) was based on the risk, injury, failure, loss of kidney function, and end-stage kidney disease (RIFLE), Acute Kidney Injury Network (AKIN), or Kidney Disease: Improving Global Outcomes criteria [11].

We compared the patients' sex, age, body mass index (BMI), first exercise class ratio, exercise duration, previous experience, medical history, initial symptom, chief complaint, and location of initial visit as clinical variables between the patient groups. Moreover, we investigated the length of hospital stay, time from activity to peak CPK, bone scan uptake, and complications. We compared the blood chemistries, such as CPK, aspartate transaminase (AST), alanine transaminase (ALT), creatine kinase-muscle/ brain (CK-MB), myoglobin, liver function test, electrolyte, blood urea nitrogen (BUN), creatinine $(\mathrm{Cr})$, osmolality, uric acid, lactate, $\mathrm{pH}$, anion gap between the groups.

\section{Statistical analysis}

The collected data were analyzed using SPSS Statistics version 22.0 (IBM Corp., Armonk, NY, USA). The chisquare test was used when the dependent variable was a categorical variable. Student's $t$-test was used when the dependent variable was continuous and followed a normal distribution, whereas Mann-Whitney test was used when the dependent variable was not. All the statistical analyses were two-sided tests, and a $p$-value less than 0.05 was considered statistically significant.

\section{RESULTS}

During the 14-year study period, 70 patients with EIR were included, but we excluded two patients with missing data and six patients with a CPK level of less than 1,500 U/L. Sixty-two patients were finally enrolled. Among the 62 patients, EIR of the $23(37.1 \%)$ patients was caused by a spinning exercise, whereas that of the 39 (62.9\%) patients was caused by other types of exercise (Fig. 1). EIR occurred in various types of exercise, including spinning $(\mathrm{n}=23)$, weight training $(\mathrm{n}=13)$, squatting $(\mathrm{n}=8)$, cycling 
$(\mathrm{n}=3)$, CrossFit $(\mathrm{n}=3)$, swimming $(\mathrm{n}=3)$, walking $(\mathrm{n}=2)$, push up $(\mathrm{n}=2)$, running $(\mathrm{n}=1)$, boxing $(\mathrm{n}=1)$, climbing $(\mathrm{n}=1)$, Pilates $(\mathrm{n}=1)$, and playing soccer $(\mathrm{n}=1)$.

The proportion of the female patients was significantly higher in the SIR group ( $78.3 \%$ vs. $38.5 \%, p=0.002$ ) (Table 1 ). The median age was not significantly different between the two groups (28.0 [22.0-30.0] vs. 26.0 [22.0-30.5], $p=0.907)$. BMI was higher in the SIR group than in the NSIR group. The first exercise class ratio was significantly higher in the SIR group than in the NSIR group $(60.9 \%$ vs. $15.4 \%, p=0.001)$. Most patients with SIR had bilateral thigh pain (91.3\% vs. $43.6 \%, p=0.001$ ), whereas those with NSIR had pain involving various muscle groups, including chest, abdomen, back, and arms. The rate of rhabdomyolysis was significantly higher in the SIR group than in the NSIR group despite the shorter exercise duration (less than 60 minutes) ( $90.5 \%$ vs. $62.9 \%, p=0.024$ ) (Fig. 2). The admission rate $(91.3 \%$ vs. $84.6 \%, p=0.523)$ and the length of hospital stay (6.0 [4.5-7.0] vs. 5.0 [3.5-6.0] days, $p=0.08)$ were higher in the SIR group. The whole-body bone scan was performed in seven and 16 patients in the SIR and NSIR groups, respectively, and bilateral thigh up- take was confirmed in seven and 13 patients, respectively.

The SIR group had more patients with a peak CPK level of 15,000 U/L or higher compared to the NSIR group (91.3\% vs. $74.4 \%, p=0.182$ ) (Fig. 2 ). The CPK value of $15,000 \mathrm{U} / \mathrm{L}$ or higher was measured at 15,000 U/L and was marked as 15,000 U/L. The laboratory test results of both the SIR and NSIR groups were as follows (Table 2): initial CPK $(15,000.0[15,000.0-15,000.0]$ vs. $15,000.0[14,839.5-$ $15,000.0] \mathrm{U} / \mathrm{L}, p=0.549)$, peak CPK $(15,000.0[15,000.0$ $15,000.0]$ vs. $15,000.0[14,839.5-15,000.0] \mathrm{U} / \mathrm{L}, p=0.359)$, AST (869.0 [632.5-1,250.5] vs. $482.0[217.5-1,333.5] \mathrm{U} / \mathrm{L}$, $p=0.117)$, and ALT (298.0 [154.0-378.0] vs. 180.0 [120.5338.0] U/L, $p=0.153)$. Each value showed a higher tendency in the SIR group. Initial CK-MB level was significantly higher in the SIR group (88.95 [25.30-103.60] vs. 25.65 [10.70-50.85] ng/mL, $p=0.012)$. However, BUN (10.60 [8.00-12.15] vs. 15.30 [11.80-18.55] mg/dL, $p<0.001), \mathrm{Cr}$ $(0.60$ [0.50-0.60] vs. $0.80[0.60-0.92] \mathrm{mg} / \mathrm{dL}, p=0.001)$, and initial $\mathrm{pH}$ (7.39 [7.37-7.40] vs. 7.42 [7.40-7.43], $p=0.042)$ were significantly lower in the SIR group.

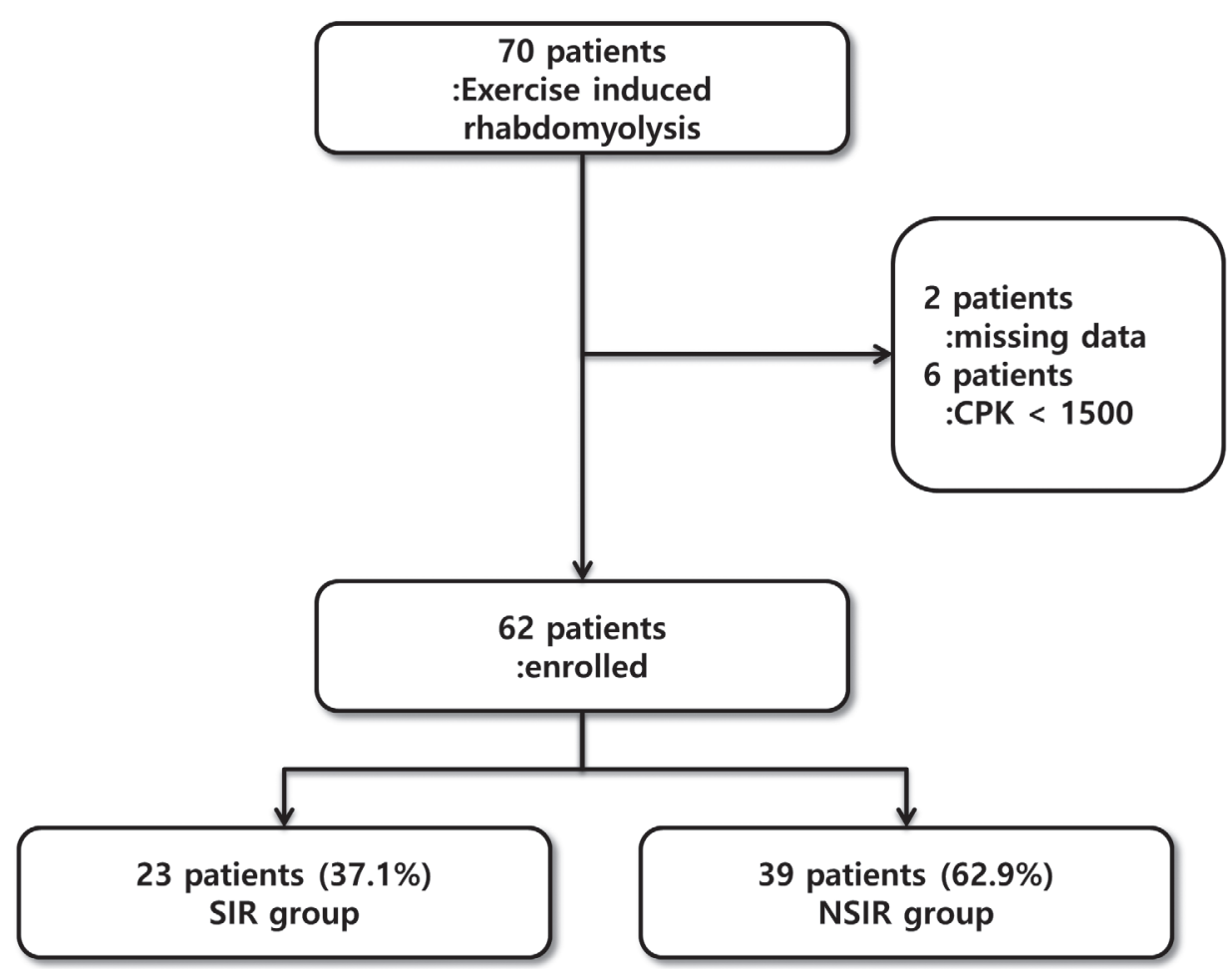

Fig. 1. Study population. CPK: creatine phosphokinase, SIR: spinning-induced rhabdomyolysis, NSIR: non-spinning exercise-induced rhabdomyolysis. 


\section{DISCUSSION}

In this study, we compared the clinical features between patients with SIR and those with NSIR. We found that SIR occurs at a higher rate during the first exercise class in women compared to NSIR, and the incidence rate is higher in SIR than in NSIR despite the short exercise duration, higher peak CPK, and longer hospital stay.

In a previous analysis, patients at risk of developing EIR tend to be younger and are usually male [12]. Moreover, it

Table 1. Comparison of clinical factors and outcomes between SIR and NSIR

\begin{tabular}{|c|c|c|c|}
\hline & $\operatorname{SIR}(n=23)$ & NSIR ( $n=39)$ & $p$-value \\
\hline Sex (female) & 18/23 (78.3) & 15/39 (38.5) & 0.002 \\
\hline Age (years) & $28.0(22.0-30.0)$ & $26.0(22.0-30.5)$ & 0.907 \\
\hline $\mathrm{BMI}\left(\mathrm{kg} / \mathrm{m}^{2}\right)$ & $24.40(21.40-26.40)$ & $22.40(20.70-24.25)$ & 0.275 \\
\hline First exercise class & $14 / 23(60.9)$ & $6 / 39(15.4)$ & 0.001 \\
\hline Most painful site (thigh) & 21/23 (91.3) & $17 / 39(43.6)$ & 0.001 \\
\hline Duration of exercise (minutes) & $60(50-60)$ & $60(60-90)$ & 0.011 \\
\hline Duration of exercise (less than 60 minutes) & 19/23 (90.5) & 22/39 (62.9) & 0.024 \\
\hline Time from activity to initial symptom (days) & $1.0(0.5-1.0)$ & $1.0(0.5-1.0)$ & 0.844 \\
\hline Time from activity to visit (days) & $3(2-4)$ & $2(1-3)$ & 0.172 \\
\hline Previous activity experience (none) & 10/11 (90.9) & $9 / 13(69.2)$ & 0.327 \\
\hline Past medical history (yes) & $2 / 23(8.7)$ & $8 / 39(20.5)$ & 0.298 \\
\hline Initial symptom & & & 1.000 \\
\hline Myalgia & $21 / 23(91.3)$ & $34 / 39(87.2)$ & \\
\hline Dark urine & $1 / 23(4.3)$ & $2 / 39(5.1)$ & \\
\hline Others & $1 / 23(4.3)$ & $3 / 39(7.7)$ & \\
\hline Chief complaint & & & 0.151 \\
\hline Myalgia & $4 / 23(17.4)$ & 13/39 (33.3) & \\
\hline Dark-urine & 19/23 (82.6) & 23/39 (59.0) & \\
\hline Others & $0 / 23(0)$ & 3/39 (7.7) & \\
\hline Location of initial visit & & & 0.055 \\
\hline $\mathrm{ED}$ & $15 / 23(65.2)$ & $34 / 39(87.2)$ & \\
\hline OPD & $8 / 23(34.8)$ & $5 / 39(12.8)$ & \\
\hline Hospital admission & 21/23 (91.3) & 33/39 (84.6) & 0.523 \\
\hline Length of hospital stay (day) & $6.0(4.5-7.0)$ & $5.0(3.5-6.0)$ & 0.080 \\
\hline Time from activity to peak CPK (day) & $3(2-4)$ & $2(1-3)$ & 0.133 \\
\hline Bone scan uptake (thigh) & $7 / 7(100.0)$ & 13/16 (81.3) & 0.526 \\
\hline \multicolumn{4}{|l|}{ Complication } \\
\hline Pulmonary edema & $0 / 23(0)$ & 1/39 (2.6) & 1.000 \\
\hline AKI & 0/23 (0) & $5 / 39(12.8)$ & 0.148 \\
\hline Hyperkalemia & $0 / 23(0)$ & 1/39 (2.6) & 1.000 \\
\hline Hemodialysis & 0/23 (0) & 1/39 (2.6) & 1.000 \\
\hline
\end{tabular}

Values are presented as number (\%) or median (interquartile range).

SIR: spinning-induced rhabdomyolysis, NSIR: non-spinning exercise-induced rhabdomyolysis, BMI: body mass index, ED: emergency department, OPD: outpatient department, CPK: creatine phosphokinase, AKI: acute kidney injury. 
has been reported that men are vulnerable to EIR because estrogen plays a protective role [13]. In addition, studies have shown that high BMI is associated with the development of rhabdomyolysis [14]. In this study, the mean age of both the SIR and NSIR groups was less than 30 years, and the age was not significantly different from the previous study. The SIR group had a higher proportion of female patients and a higher BMI than the NSIR group, which might be possibly due to the following reasons: rel-

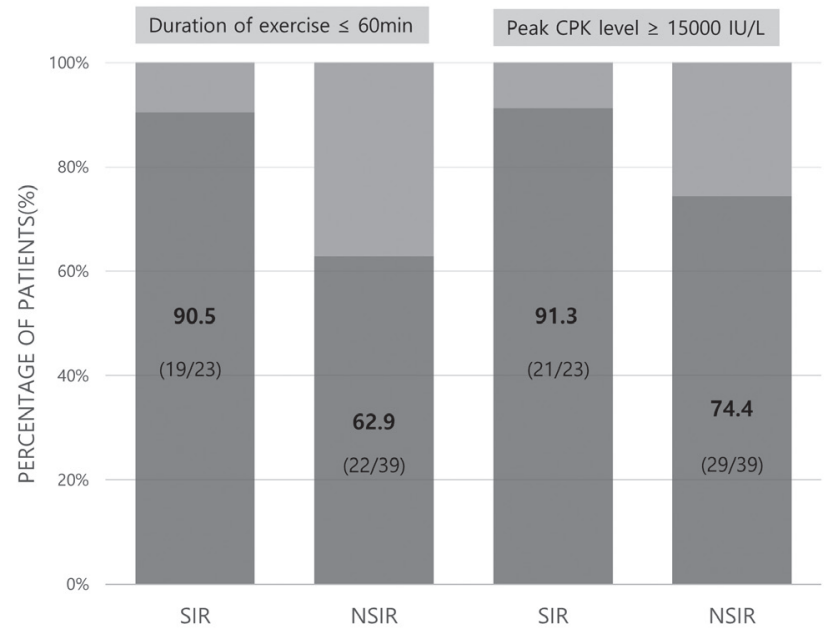

Fig. 2. Comparison of exercise duration and peak CPK level between SIR and NSIR. CPK: peak creatine phosphokinase, SIR: spinning-induced rhabdomyolysis, NSIR: non-spinning exercise-induced rhabdomyolysis. atively overweight women are performing more spinning exercises than other exercises; they had a nutritional imbalance, or the majority of patients have never exercised before [7].

Spinning class participants adopt different positions and vary the resistance on the cycle, thus simulating climbing hills or cycling on flat roads. This requires repetitive use of the large muscle groups of the bilateral hip and legs, mainly the gluteus maximus and quadriceps muscles [15]. In this study, the majority of patients with SIR complained of thigh pain for the abovementioned reasons, and all patients who underwent a bone scan had thigh uptake.

EIR is characterized by the breakdown and necrosis of striated skeletal muscles after a physical activity. This muscle injury leads to the leakage of muscle cell contents, including electrolytes, myoglobin, and other sarcoplasmic proteins (e.g., creatinine phosphokinase, aldolase, lactate dehydrogenase, alanine aminotransferase, and aspartate aminotransferase) into the circulation [16]. In particular, serum CPK begins to increase 2-12 hours after the adjustment period and peaks within 24-72 hours. CPK has a half-life of 1.5 days and its removal from the plasma is relatively late; thus, it is likely to remain high at the time of the test, making it more sensitive for muscle injury determination and diagnosis of rhabdomyolysis

Table 2. Comparison of the laboratory test results between SIR and NSIR

\begin{tabular}{|lccc|}
\hline \multicolumn{1}{|c|}{ SIR $(\mathbf{n}=\mathbf{2 3})$} & NSIR $(\mathbf{n}=\mathbf{3 9})$ & \multicolumn{1}{c|}{$\boldsymbol{p}$-value } \\
\hline CPK (U/L) & & & \\
Peak & $15,000.0(15,000.0-15,000.0)$ & $15,000.0(14,839.5-15,000.0)$ & 0.359 \\
Initial & $15,000.0(15,000.0-15,000.0)$ & $15,000.0(14,839.5-15,000.0)$ & 0.549 \\
Discharge & $3,347.0(1,750.5-7,005.5)$ & $3,084.0(2,049.5-6,717.0)$ & 0.901 \\
CKMB (initial, ng/mL) & $88.95(25.30-103.60)$ & $25.65(10.70-50.85)$ & 0.012 \\
Myoglobin (initial, ng/mL) & $1,000(1,000-1,000)$ & $1,000(1,000-1,000)$ & 0.155 \\
AST (U/L) & $869.0(632.5-1,250.5)$ & $482.0(217.5-1,333.5)$ & 0.117 \\
ALT (U/L) & $298.0(154.0-378.0)$ & $180.0(120.5-338.0)$ & 0.153 \\
BUN (mg/dL) & $10.60(8.00-12.15)$ & $15.30(11.80-18.55)$ & $<0.001$ \\
Cr (mg/dL) & $0.60(0.50-0.60)$ & $0.80(0.60-0.92)$ & 0.001 \\
pH & $7.39(7.37-7.40)$ & $7.42(7.40-7.43)$ & 0.042 \\
\hline
\end{tabular}

Values are presented as median (interquartile range).

SIR: spinning-induced rhabdomyolysis; NSIR: non-spinning exercise-induced rhabdomyolysis, CPK: creatine phosphokinase, CKMB: creatine kinase-muscle/ brain, AST: aspartate transaminase, ALT: alanine transaminase, BUN: blood urea nitrogen, Cr: creatinine. 
[17]. In this study, the SIR group had a higher CPK level and longer hospital stay than the NSIR group. Moreover, the incidence rate is higher in patients with SIR than in those with NSIR despite the shorter exercise duration (less than 1 hour). Given that, unlike other exercises, the spinning classes have fixed start and end times, which are accompanied by some force regardless of the individual's basic exercise ability. In addition, it is presumed that a competition intensity is stimulated because majority of the patients perform spinning exercises at the same time; thus, muscle damage is easily caused by short exercise duration with a high exercise intensity. Therefore, in the first class, a step-by-step exercise program is recommended, avoiding full intensity spinning. The results showed a higher CPK level and a longer hospital stay in SIR than in NSIR. But these results were not statistically significant and probably due to the small sample size. In general, the spinning classes take a fixed duration, and usually last for 40-60 minutes [8]. Since the study about spinning exercise duration has not been performed, further studies are required to compare the results about the differences of exercise duration.

Studies have shown that CK-MB can be a sensitive indicator of skeletal muscle injury in EIR [18]. In this study, the initial CK-MB level of SIR was significantly higher than that of NSIR, which suggests that muscle injury was more severe in SIR than in NSIR.

The most relevant complication of rhabdomyolysis is AKI due to myoglobin deposits in the renal tubules, leading to direct toxicity, intrarenal vasoconstriction, and decreased glomerular filtration rate [19]. The reported incidence of AKI in patients with rhabdomyolysis ranges from $13 \%$ to $50 \%[1,20]$, and the overall mortality of AKI is $8 \%$ [21]. A study found that EIR had a lower AKI rate than other etiologies [1]. McMahon et al. [22] reported that the rates of renal replacement therapy and in-hospital mortality were lower in EIR than in rhabdomyolysis with other etiologies. One study reported that 1 of 14 SIR cases and 0 of 15 NSIR cases developed AKI [20]. In our study, none of the 23 SIR patients developed AKI, whereas 5 of the 39 NSIR patients developed AKI. This low rate of renal failure in the SIR group is consistent with the studies of Hernández-Contreras et al. [2] and Montero et al. [23]. We found that the SIR group had a higher CPK level, lower Cr level, and lower incidence rate of AKI development than the NSIR group. The reason for this was that most of the young women in the SIR group were healthy individuals without any past medical history, which may have affected the low $\mathrm{Cr}$ level and the low incidence of AKI. One of the five patients with AKI in the NSIR group underwent hemodialysis. This 19-year-old woman was admitted due to myalgia and oliguria after performing squatting exercises and was discharged after hemodialysis due to oliguria, hyperkalemia, and generalized edema during hospitalization.

In this study, no prominent acidosis was found in both the SIR and NSIR groups, but the initial pH was lower in the SIR group $(p=0.042)$. Acute metabolic acidosis is another complication of rhabdomyolysis. Acidosis results from the release of intracellular phosphate and sulfate. Sulphur-containing proteins released in large amounts can lead to hydrogen and sulphate loads that overwhelm renal excretory mechanisms. Other causes of acidosis in rhabdomyolysis include lactic acidosis from ischemia and acidosis from uremia [24]. Therefore, the lower $\mathrm{pH}$ of patients with SIR in our study may indicate that the muscle injury or ischemia is more severe in SIR than in NSIR; thus, the amount of intracellular sulphate exceeds the renal excretory mechanism.

In our study, the incidence rate is higher in SIR than in NSIR despite the short exercise duration. There are specific guidelines to prevent SIR. First, sufficient amount of time to warmup should be considered. Second, standard education on SIR is also important. Instructors and participants should understand the danger, symptoms, and signs of SIR. Third, exercise should be modified or abstained in the presence of evitable risk factors such as viral diseases. Lastly, sufficient hydration and nutrition support can be effective [4].

This study had several limitations. First, this study was retrospective in nature, was conducted in a single center, and had a small sample. In addition, accurate values could not be compared when the upper limit was exceeded, since the upper limits of CPK and myoglobin level were $15,000 \mathrm{U} / \mathrm{L}$ and $1,000 \mathrm{ng} / \mathrm{mL}$, respectively. Elevation of $\mathrm{CPK}$ is one of the main serologic criteria defining the disease entity. However, there is no definite level of the CPK increase that could identify clinically relevant EIR [25]. In 
addition, the bone scan was not performed on many cases in this study. Therefore, prospective large-scale studies should be conducted in the future.

\section{CONCLUSION}

SIR occurs at a higher rate during the first exercise class in women compared to NSIR, and the incidence rate is higher in patients with SIR than in those with NSIR despite the shorter exercise duration (less than 1 hour). Compared with NSIR patients, most patients with SIR complained of thigh pain during admission to the hospital. It is necessary to recognize these risks during spinning exercises and to perform the exercises sequentially and systematically. Moreover, instructors should be aware of the possibility of rhabdomyolysis in spinning exercise classes. Our study has small sample size, so large-scale studies will be needed.

\section{REFERENCES}

1. Melli G, Chaudhry V, Cornblath DR. Rhabdomyolysis: an evaluation of 475 hospitalized patients. Medicine (Baltimore) 2005;84:377-87.

2. Hernández-Contreras ME, Torres-Roca M, Hernández-Contreras V, Rosa Salazar V, Mar García-Méndez M, García-Pérez B, et al. Rhabdomyolysis after initial session of indoor cycling: analysis of 11 patients. J Sports Med Phys Fitness 2015;55:1371-5.

3. Tonin P, Lewis P, Servidei S, DiMauro S. Metabolic causes of myoglobinuria. Ann Neurol 1990;27:181-5.

4. Kim J, Lee J, Kim S, Ryu HY, Cha KS, Sung DJ. Exercise-induced rhabdomyolysis mechanisms and prevention: a literature review. J Sport Health Sci 2016;5:324-33.

5. Cutler TS, DeFilippis EM, Unterbrink ME, Evans AT. Increasing incidence and unique clinical characteristics of spinning-induced rhabdomyolysis. Clin J Sport Med 2016;26:429-31.

6. Rao A. Exercise-induced exertional rhabdomyolysis. The Southwest Respiratory and Critical Care Chronicles 2018;6:12-6.

7. Kim D, Ko EJ, Cho H, Park SH, Lee SH, Cho NG, et al. Spinning-induced rhabdomyolysis: eleven case reports and review of the literature. Electrolyte Blood Press 2015;13:58-61.

8. Kim YH, Ham YR, Na KR, Lee KW, Choi DE. Spinning: an arising cause of rhabdomyolysis in young females. Intern Med J
2016;46:1062-8.

9. Martínez JJ, Oliveira CL, Meneses AL, Rodríguez SA, Corrales PP, López AH, et al. Rhabdomyolysis due to primary hyperaldosteronism. Endocrinol Nutr 2009;56:431-4.

10. Gabow PA, Kaehny WD, Kelleher SP. The spectrum of rhabdomyolysis. Medicine (Baltimore) 1982;61:141-52.

11. Lopes JA, Jorge S. The RIFLE and AKIN classifications for acute kidney injury: a critical and comprehensive review. Clin Kidney J 2013;6:8-14.

12. Alpers JP, Jones LK Jr. Natural history of exertional rhabdomyolysis: a population-based analysis. Muscle Nerve 2010;42:487-91.

13. Clarkson PM, Hubal MJ. Are women less susceptible to exercise-induced muscle damage? Curr Opin Clin Nutr Metab Care 2001;4:527-31.

14. Parmar S, Chauhan B, DuBose J, Blake L. Rhabdomyolysis after spin class? J Fam Pract 2012;61:584-6.

15. Brogan M, Ledesma R, Coffino A, Chander P. Freebie rhabdomyolysis: a public health concern. Spin class-induced rhabdomyolysis. Am J Med 2017;130:484-7.

16. Bosch X, Poch E, Grau J. Rhabdomyolysis and acute kidney injury. N Engl J Med 2009;361:62-72.

17. Kang SW, Kim YW, Kim YH. Analysis of nontraumatic rhabdo myolysis during recent 2 years. Korean J Med 2004;67:467-74.

18. Siegel AJ, Silverman LM, Holman BL. Elevated creatine kinase MB isoenzyme levels in marathon runners. Normal myocardial scintigrams suggest noncardiac source. JAMA 1981;246:2049-51.

19. Petejova N, Martinek A. Acute kidney injury due to rhabdomyolysis and renal replacement therapy: a critical review. Crit Care 2014;18:224.

20. Holt SG, Moore KP. Pathogenesis and treatment of renal dysfunction in rhabdomyolysis. Intensive Care Med 2001;27:803-11.

21. Benish M, Zeitlin I, Deshet D, Beigel Y. Massive indoor cycling-induced rhabdomyolysis in a patient with hereditary neuropathy with liability to pressure palsy. Isr Med Assoc J 2012;14:712-4.

22. McMahon GM, Zeng X, Waikar SS. A risk prediction score for kidney failure or mortality in rhabdomyolysis. JAMA Intern Med 2013;173:1821-8.

23. Montero J, Lovesio C, Godoy MV, Ruiz G. Rhabdomyolisis caused by spinning in nine patients. Medicina (B Aires) 2009;69 (1 Pt 2):153-6.

24. Khan KY. Rhabdomyolysis: a review of the literature. Neth J Med 2009;67:272-83.

25. Lee G. Exercise-induced rhabdomyolysis. R I Med J (2013) 2014;97:22-4. 